\title{
Dense flow field interpolations from PTV data in the presence of generic solid boundaries
}

\author{
Bora O. Cakir ${ }^{1,2 *}$, Gabriel Gonzalez ${ }^{2}$, Andrea Sciacchitano ${ }^{2}$, Bas van \\ Oudheusden $^{2}$ \\ ${ }^{1}$ von Karman Institute for Fluid Dynamics, Turbomachinery and Propulsion Department, \\ Sint-Genesius-Rode, Belgium \\ ${ }^{2}$ Delft University of Technology, Faculty of Aerospace Engineering, Delft, The Netherlands \\ *bora.orcun.cakir@vki.ac.be
}

\begin{abstract}
Three-dimensional flow measurements by Particle Tracking Velocimetry (PTV) provide scattered flow information, that often needs to be interpolated onto a regular grid. Therefore, the use of experimental data assimilation approaches such as VIC+ (Schneiders and Scarano, 2016) were proposed to enhance the instantaneously available spatial resolution limits beyond that of the PTV measurements. Nevertheless, there exists no prior attempt to perform the data assimilation when the flow is in direct contact with physical objects. Thus, in order to handle generic solid body intrusions within the flow fields of VIC+ application, the utilization of Arbitrary Lagrangian-Eulerian and immersed boundary treatment approaches of the computational fluid-structure interaction (FSI) frameworks are proposed. The introduced variants over the standard VIC+ are assessed with a high fidelity numerical test case of flow over periodic hills. The accuracy superiority of the flow field reconstructions with the proposed approaches are denoted especially in close proximity of the interaction surface. An experimental application of the introduced methods is demonstrated to compute the pressure distribution over an unsteadily moving elastic membrane surface, revealing the time-resolved interaction between the flow structures and the membrane deformations.
\end{abstract}

\section{Introduction}

Tomographic PIV (Tomo-PIV) (Elsinga et al., 2006) allows acquisition of flow information over a three dimensional measurement domain. However, Tomo-PIV measurements are accompanied by stringent limitations on the maximum achievable measurement volume due to the dispersed intensity of light illumination, limited light scattering performance of tracer particles and resolution characteristics of recorded images (Tokarev et al., 2013). Accordingly, introductory applications of Tomo-PIV were performed in a volume of $13 \mathrm{~cm}^{3}$, while the maximum measurement volume size achieved with micrometric tracer particles is documented to be $6 \times 22 \times 8 \mathrm{~cm}^{3}$ where the acceptable signal-to-noise ration distribution already limited the effective depth to $5 \mathrm{~cm}$ (Fukuchi, 2012). Hence, in order to enable large scale applications of Tomo-PIV experiments, the use of neutrally buoyant Helium Filled Soap Bubbles (HFSB) as tracers for PIV experiments was proposed (Scarano et al., 2015; Caridi et al., 2016). Their controllable larger size and density, provided a great opportunity of achieving suitable flow tracing qualities and superior light scattering characteristics.

Nevertheless, exploiting large scale PTV techniques, the limited production rate and seeding rate of the HFSB restrict the concentration of tracer particles within the measurement domain which in turn alleviates the instantaneously available spatial resolution. Thus, the reliability and accuracy of volumetric reconstruction might be compromised. Considering the spatial scarcity of HFSB particles, employing a particle tracking approach enables a greater level of accuracy in terms of determining particle location, velocity and acceleration. However, the data obtained from PTV results in a scattered formation over the measurement domain. Although linear interpolation of scattered data might resolve the problem of increasing the spatial resolution of fluidic information, the independency of this approach from statistical or physical characteristics of the flow in measurement would comprise significant numerical errors that results in incoherent and unphysical flow structures. 
Therefore, advanced interpolation methods are required for increasing the spatial resolution of the available experimental data which would provide greater accuracy and physical coherence of the dense flow field information. Accordingly, multiple interpolation frameworks were developed based on the statistical formulations of various spatial averaging and data fitting approaches. The Adaptive Gaussian Windowing (AGW) proposed by Agui and Jimenez (1987), captures the scattered information in a measurement volume on a predefined grid using a Gaussian weight averaging over the windows of Euclidean distances defined with respect to the grid location of interest. Furthermore, Gaussian windowing is further elaborated on using Gaussian radial basis functions (RBF) in which Casa and Krueger (2013) utilized an iterative optimization procedure to obtain the best fit of data interpolation on a grid structure with respect to the original scattered data. On the other hand, more convoluted approaches in the context of incorporating the physical description of fluid dynamics to the interpolation procedure. (Gesemann et al., 2016) introduced the use of 3D cubic Bsplines for increasing signal-to-noise ratio of particle information reconstruction over particle velocity and accelerations. The method includes two main steps referred to as TrackFit and FlowFit. TrackFit initiates the reconstruction algorithm with a noise reduction of the particle intensity signals. Then, 3D B-splines are calculated for velocity and acceleration (or pressure) information by minimizing a cost function while enforcing divergence free field constraints for incompressible flows.

Another application of flow physics based experimental data assimilation is introduced by Schneiders et al. (2015) where the temporal information is used to enrich the spatial information for elevated accuracy of flow field reconstruction at higher spatial resolution levels. The resultant VIC+ method, utilizes the Vortexin-Cell (VIC) model presented by Christiansen (1973) for coupling vorticity and velocity field information of the fluid of interest enabling the representation of the fluid behavior on a global sense over the prescribed computational domain, provided that appropriate boundary conditions are imposed. Moreover, the vorticity transport equation allows the computation of local time derivative component for the Lagrangian acceleration term only by means of velocity and vorticity fields via eliminating the contribution of pressure gradients. Hence, VIC+ also provides an opportunity to increase the resolution of material derivative information as its optimization procedure is based on maximizing the proximity of not only the velocity vectors to the measured values but also the accelerations (Schneiders and Scarano, 2016).

Although VIC+ and its variants are proven to be successfully increasing the spatial resolution of flow field information using scattered measurement data on uniformly structured computational grids, their applicability is restricted to purely fluidic domains. However, most practical engineering applications are influenced by dynamic aeroelastic behaviors which contain non-uniform solid boundaries that might deform unsteadily owing to their interaction with the fluid medium. Thus, computation of fluid information in presence of curved walls or solid objects (stationary or moving) introduces significant drawbacks in terms of numerical accuracy and appropriate surface definition for implementation of adequate boundary conditions. Without proper definition of those solid boundaries, the accuracy of determining the corresponding fluid behavior is further downgraded as the information transfer between fluid and solid domains strongly depend on the interface description where the surface loading exerted by the flow and the boundary shape are in an interactive relationship (Dowell, 2004). More recently proposed methods for managing non-uniform solid intrusions, separate the computational domain in to multiple regions in which close surface locations are handled by varying the integration direction. Although these methods provide accurate results in comparison with the extrapolation techniques, the improvements are associated with increased levels of complexity and computational cost. Therefore the current study introduces two approaches for providing VIC+ algorithm with the capability of performing data assimilation in presence of solid boundaries by implementing the well known computational FSI frameworks of Arbitrary Lagrangian-Eulerian (Noh, 1963) and immersed boundary treatment methods (Peskin, 1982).

\section{Methodology}

Two main approaches, named Arbitrary Lagrangian-Eulerian VIC+ (ALE-VIC+) and Immersed Boundary $\mathrm{VIC}+(\mathrm{ImVIC}+)$, are proposed to handle the solid boundary effects for VIC+ applications. Boundary fitted coordinate systems and mesh adaption procedures are employed in ALE-VIC+ approach, whereas in ImVIC+ method the immersed boundary treatment is utilized to satisfy the appropriate boundary conditions while preserving uniform mesh formations. 


\subsection{Arbitrary Lagrangian-Eulerian approach for VIC+}

In order to avoid the individual shortcomings of Eulerian and Lagrangian perspectives while leveraging from their respective advantages, a technique referred to as the Arbitrary Lagrangian-Eulerian (ALE) method is introduced by Noh (1963), where both approaches are utilized in a coupled manner to handle both fluidic and structural domains. As the necessity of a coupled method emerges from the motion of boundaries enclosing the fluidic domain, the corresponding mesh structures are required be modified accordingly. Hence, a natural approach can be considered as completely regenerating the grid structure at each time step. However, this process generally requires considerable user interaction and immense computational resources (Luke et al., 2012). Instead, grid deformation algorithms provide a valuable solution categorized under two main groups of physical analogy based (Farhat et al., 1998; Löhner and Yang, 1996; Helenbrook, 2003) and interpolation based schemes (Wang and Przekwas, 2012; Jones and Samareh-Abolhassani, 2012; Liu et al., 2006). Utilizing these various methodologies, there exists multiple applications of the ALE method for vortex simulations based on the VIC model, for cost-effective high-fidelity numerical simulations of fluidstructure interaction (FSI) problems. In this regard, Cottet and Poncet (2004) performed conformal mapping of the fluidic domain for three dimensional direct numerical simulations of wall bounded flows where the grid structure in close proximity of a sphere was fitted to the surface of the solid object for application of a hybrid particle-mesh vortex method. Furthermore, Kudela and Kozlowski (2009) employed a boundary fitted coordinate system for flow simulations around arbitrary shaped objects using the VIC framework during which fourth order interpolation kernels used by Cottet and Koumoutsakos (2000); Sagredo and Tercero (2003) are modified for particle-mesh switching of vorticity distributions in the near wall regions.

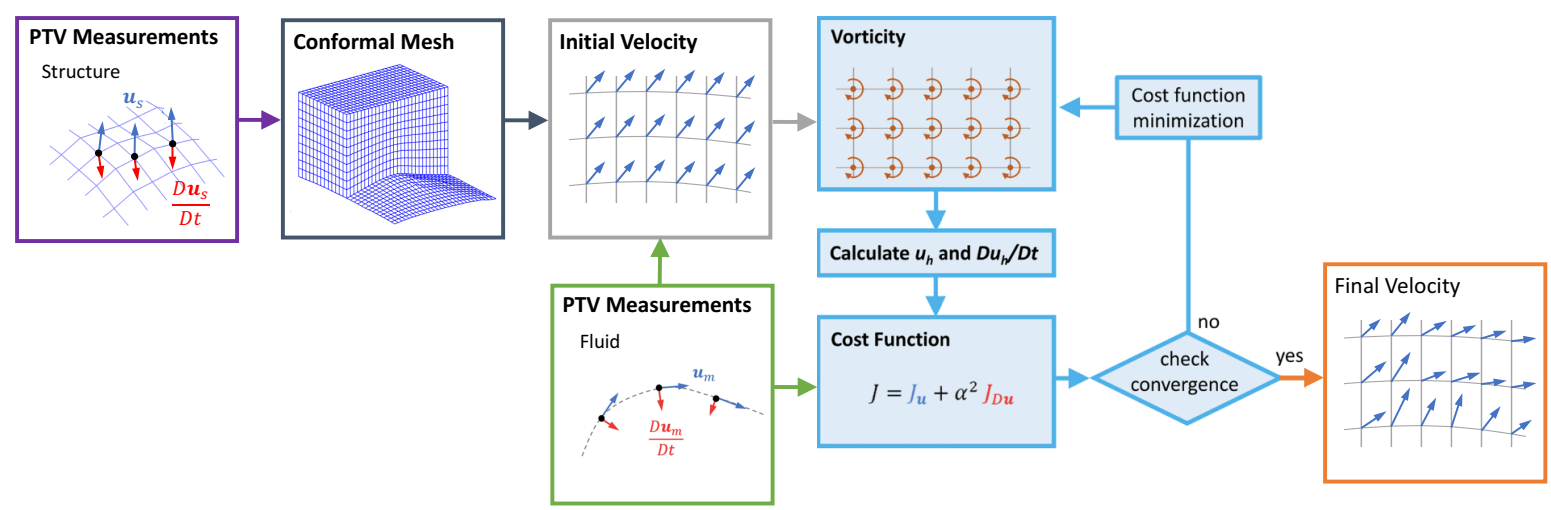

Figure 1: ALE-VIC+ reconstruction framework. The PTV measurements for the structural component is provided (purple box). The rectangular structured computational grid is deformed for boundary fitted coordinate generation (black box). The PTV measurements for the flow is provided (green box). An initial velocity estimate is made (gray box), which is input into VIC+ iterative procedure (blue boxes) to find the optimization variables that yield a velocity and material derivative distributions of minimum discrepancy with the PTV measurements (orange box).

Employment of boundary fitted coordinate systems and their deformations for treating unsteadily deforming domains for VIC+ applications is composed of two main steps; a computational grid is generated according to the surface information and the flow governing equations within VIC+ framework are solved over the generated mesh structure. The algorithm is initiated by generating a conformal grid structure from the boundary shape definitions either known a priori or captured by means of optical measurement methods. After an initial boundary fitted grid structure is generated, the mesh is deformed according to the boundary motion so that the exact interaction between the fluidic and solid domains can be expressed in a time-resolved manner. In order to achieve a continuous boundary conformation, the choice of deformation scheme is determined as RBF based mesh deformations (de Boer et al., 2007) due to their superior characteristics of mesh deformation accuracy and cost-efficiency (Smith et al., 2000, Beckert and Wendland, 2001). The formulations employed for RBF based mesh deformation method utilized are described by (de Boer et al., 2007) where a comparitive study over many variants of RBFs was conducted using two different mesh quailty metrics based on mesh size preservation and mesh skewness. Among 14 different RBFs with compact and global supports, Thin Plate Spline (TPS) and CP $C^{2}$ RBFs stood out both in terms of keeping 
the size alterations and skewness to a minimum whilst not compromising mesh adaptation efficiency. According to the presented results, $\mathrm{CP} C^{2} \mathrm{RBF}$ is utilized to update the computational grid due to a superior performance of mesh deformations especially in close proximity of the immersed boundary surface.

Then, the flow governing equations on the updated conformal grid are solved by performing a oneto-one mapping between the physical and computational coordinate systems. The mapping relates to the transformation variables necessary for accurate description of mathematical operators constructing the link between the two grids. The physical coordinate system is selected as a Cartesian one since the flow field properties are obtained from the PIV/PTV measurements are already defined on a Cartesian coordinate system. Hence, the computational grid locations in any mesh form can be expressed as functions of the physical coordinate system variables,

$$
x_{C}=x_{C}\left(x_{P}, y_{P}, z_{P}\right) \quad y_{C}=y_{C}\left(x_{P}, y_{P}, z_{P}\right) \quad z_{C}=z_{C}\left(x_{P}, y_{P}, z_{P}\right)
$$

where subscript $C$ and $P$, refer to the physical and computational coordinates respectively. Accordingly, the transformation variables defining the link between the two coordinate systems is represented by means of a Jacobian matrix. Therefore, the flow governing equations within VIC+ method are described on the computational coordinates by transforming the vector variables utilizing the chain rule and the transformation matrix.

$$
\mathbf{J}_{T}=\frac{\partial\left(x_{C}, y_{C}, z_{C}\right)}{\partial\left(x_{P}, y_{P}, z_{P}\right)}=\left[\begin{array}{lll}
\frac{\partial x_{C}}{\partial x_{P}} & \frac{\partial x_{C}}{\partial y_{P}} & \frac{\partial x_{C}}{\partial z_{P}} \\
\frac{\partial y_{C}}{\partial x_{P}} & \frac{\partial y_{C}}{\partial y_{P}} & \frac{\partial y_{C}}{\partial z_{P}} \\
\frac{\partial z_{C}}{\partial x_{P}} & \frac{\partial z_{C}}{\partial y_{P}} & \frac{\partial z_{C}}{\partial z_{P}}
\end{array}\right]
$$

Finally, as the orientation of velocity and acceleration vectors are preserved on the Cartesian descriptions, the resultant flow properties of velocity and material acceleration values are linearly interpolated at the original particle locations for calculating the error between the dense flow field interpolation and the measurement data. Following the exact procedure of adjoint gradient computation introduced for VIC+, additional modifications are implemented to take into account the vector transformations between the physical and computational grid structures. Hence, the gradients for the proceeding steps of the optimization are calculated and the optimization procedure is performed until a specified convergence criteria is achieved.

\subsection{Immersed boundary treatment approach for VIC+}

The VIC framework introduced by Christiansen (1973) allows the fast Fourier Transform (FFT) based Poisson solvers to be employed over a predefined computational grid (Wu and JaJa, 2013) allowing higher fidelity and resolution capabilities, while preserving the computational efficiency. As the fast Poisson solvers are utilized to characterize the rotational component of velocity vectors, physical intrusions within the flow field are required to be handled employing additional velocity or forcing terms (Peskin, 1982). Therefore, the need for boundary fitted coordinate systems and introducing transformation operations between computational and physical coordinate systems is obliterated. Accordingly, Walther and Morgenthal (2002) and Cottet and Poncet (2004) implemented integral boundary equations on the VIC method in order to impose no-through and no-slip boundary conditions by means of surface singularities. However, defining no-through boundary condition over the penetrating velocity components results in an integral boundary equation which corresponds to an ill-posed Fredholm integral equation of first kind. Instead, the vortex sheet strengths are determined via the tangential velocity components yielding a Fredholm integral equation of second kind (Beale and Greengard, 1994). Furthermore, in order to obtain a unique solution for multiply connected regions, Kelvins theorem of circulation conservation or Kutta condition is introduced as an additional constraint (Morgenthal and Walther, 2007).

Implementation of the immersed boundary treatment for VIC+ method is based on the theory of vector decomposition provided via the Helmholtz theorem where the rotational component obtained from the VIC method is superimposed with the potential flow component computed from the immersed boundary corrections. The numerical implementation of boundary integral equations for adequate description of boundary conditions over the interface surfaces is performed using the panel method introduced by Hess and Smith (1967). Considering that the structural information is obtained by means of either one of the aforementioned approaches described for ALE-VIC+, the solid boundary surface is characterized by means of quadrilateral panels each equipped with singularity elements of sources and doublets to introduce a scalar potential influence of the physical intrusion. With singularity elements attached, the potential inductions of the quadrilateral panels are computed over the complete computational domain employing the formulations provided by Katz and Plotkin (2001). 


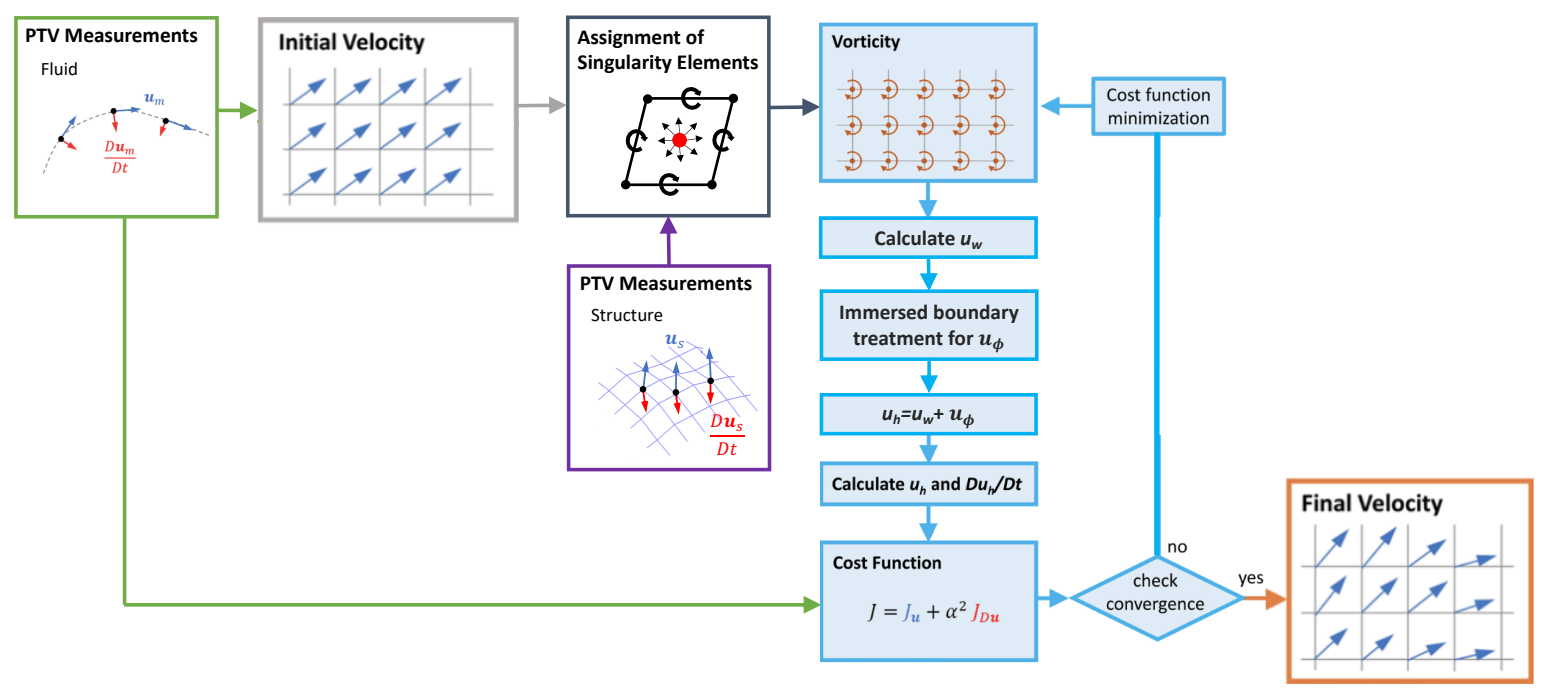

Figure 2: ImVIC+ reconstruction framework. The PTV measurements for the flow is provided (green box). An initial velocity estimate is made (gray box). The PTV measurements for the structural component is provided (purple box). Surface elements are assigned with singularities (black box). The resultant velocity fields is used as an input into VIC+ iterative procedure while velocity fields are corrected with immersed boundary treatment (blue boxes) to find the optimization variables that yield velocity and material derivative distributions of minimum discrepancy with the PTV measurements (orange box).

Initializing with a freestream velocity value, the corresponding vorticity strengths are utilized to compute the velocity distribution over the computational domain from the VIC method. The calculated velocity field penetrates through the physical boundaries where the normal velocity components to each panel are equated to the relative source strengths $\left(\sigma_{i}\right)$ of the corresponding panels in order to reduce the strengths of the dipoles $\left(\mu_{i}\right)$ for ensuring numerical uniqueness (Tarafder et al., 2010).

$$
\sigma_{i}=\mathbf{n}_{i} \cdot \mathbf{V}_{i}
$$

Furthermore, since the self induced scalar potential should vanish at collocation points over a surface of singularities (Lewis, 1991), the dipole strengths are calculated by constructing a linear system of equations that total sum of potential induction at the central locations of the quadrilateral panels equals to zero.

$$
\sum_{i=1}^{N} a_{i} \mu_{i}+\sum_{i=1}^{N} b_{i} \sigma_{i}=0
$$

In order to establish a unique solution, the corresponding vortex rings (dipoles) are imposed to comply with the conversation of circulation while their strengths are being determined. The resultant overdetermined linear system is solved employing a least squares method (Soifer, 2013). Moreover, the scalar potential field induced by the surface singularities is differentiated in there dimensions to obtain the velocity vector components,

$$
\mathbf{u}_{\phi}=\left\langle u_{\phi}, v_{\phi}, w_{\phi}\right\rangle=\left\langle\frac{\partial \phi}{\partial x}, \frac{\partial \phi}{\partial y}, \frac{\partial \phi}{\partial z}\right\rangle
$$

which are superimposed with the rotational velocity components to calculate the resultant velocity field distributions.

$$
\mathbf{u}=\mathbf{u}_{\omega}+\mathbf{u}_{\phi}
$$

Then, the corresponding velocity and vorticity fields are utilized to calculate the material derivative distributions over the computational domain in accordance to the inviscid Navier-Stokes formulation. Finally, the cost function for the optimization procedure is determined over the error accumulation of velocity and 
material acceleration values at the original particle track locations in comparison to the measured data. For the optimization procedure, it is assumed, due to the dependence of the potential flow component to the rotational elements, that the errors directly relate to the Vortex-in-Cell base which is dictated by the vorticity distributions. Hence, for each step of the optimization procedure, the gradient is calculated in terms of the vorticity strengths and the corresponding potential flow component is calculated to correct the velocity field distributions in order to satisfy the physical boundary condition of no penetration through the solid surfaces.

\section{Numerical Assessment}

Validation studies of the proposed methods are performed with a direct numerical simulation (DNS) of flow over periodic hills (Chen et al., 2014). The numerical simulations are performed with periodic boundary conditions connecting the inflow and outflow boundary conditions while the non-uniform surface contours of the hills are treated by means of an immersed boundary method to account for their influence over the fluidic domain. The inflow conditions are initiated with a non-dimensional uniform velocity distribution of $u=1$ which corresponds to a hill height based Reynolds number of $R e_{h}=10,595$ as the non-dimensional kinematic viscosity is prescribed to be $v=9.45 \times 10^{-5}$.
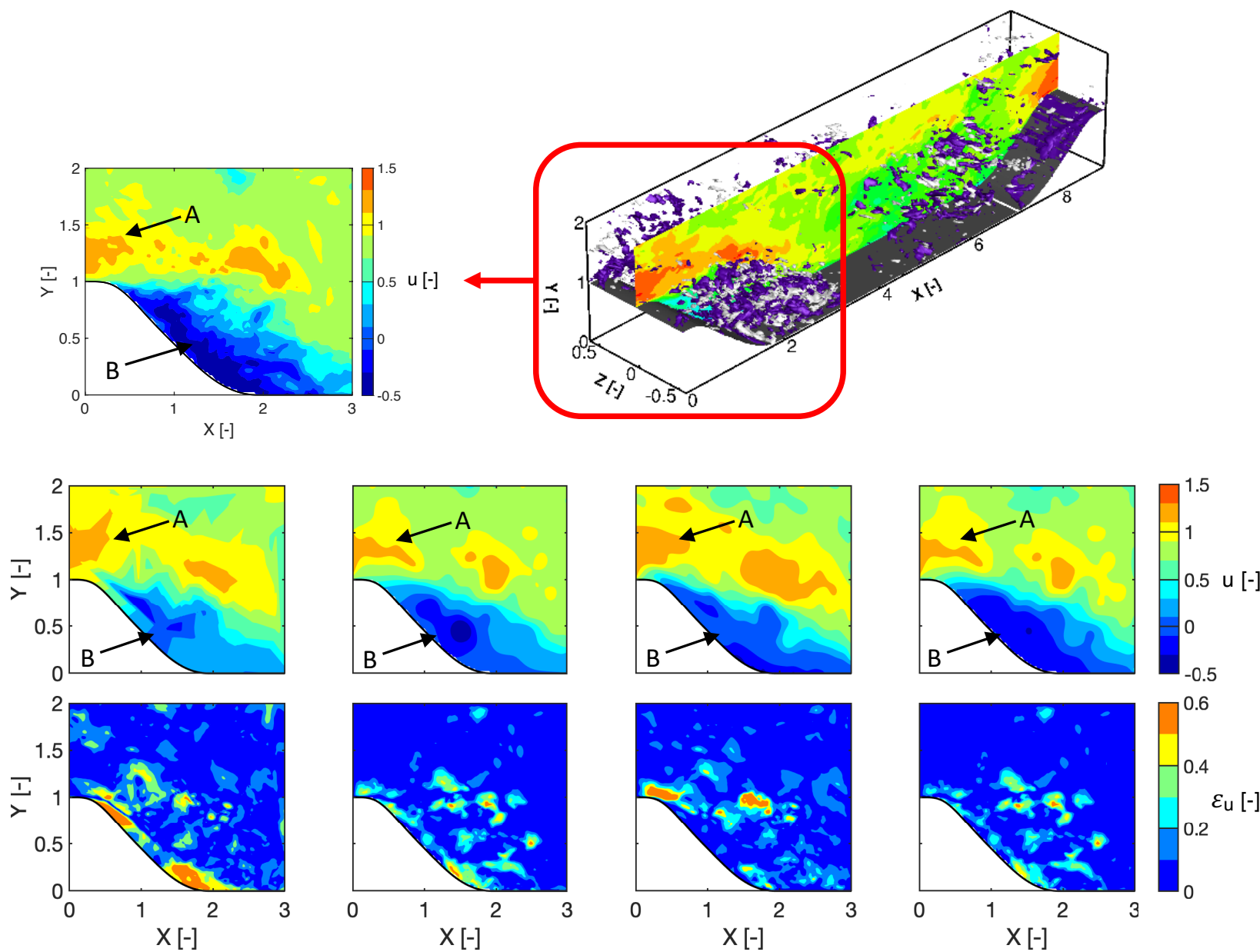

Figure 3: Instantaneous non-dimensional streamwise velocity distribution over the $Z=0$ plane with nondimensional streamwise vorticity isosurfaces of $\omega_{x}=-10$ (purple) and $\omega_{x}=10$ (white) for the reference DNS results $\left(I^{\text {st }}\right.$ row). Instantaneous non-dimensional streamwise velocity distribution over the $Z=0$ plane with tri-linear interpolation, VIC,$+ A L E-V I C+$ and ImVIC $+\left(2^{\text {nd }}\right.$ row, from left to right $)$. Planar distributions of non-dimensional streamwise velocity reconstruction error magnitudes over the $Z=0$ plane with tri-linear interpolation, VIC+, ALE-VIC+ and ImVIC+ (3rd row, from left to right $)$. 
In order to provide an accurate representation of scattered particle track information, the DNS results are randomly downsampled with particle track concentrations of $C=10 \mathrm{part} / h^{3}$. The corresponding particle image density over the projected volumes obtained as $N_{P}=0.012 \mathrm{ppp}$ aiming slightly below the particle concentrations obtained during the experimental campaigns in Section 4. Stimulation of PTV data structure is achieved via a pseudo-particle tracking approach using a Runge-Kutta 4 time integration procedure (Hwang et al., 2007). In accordance to the documented track lengths by Schanz et al. (2016) for accurate extraction
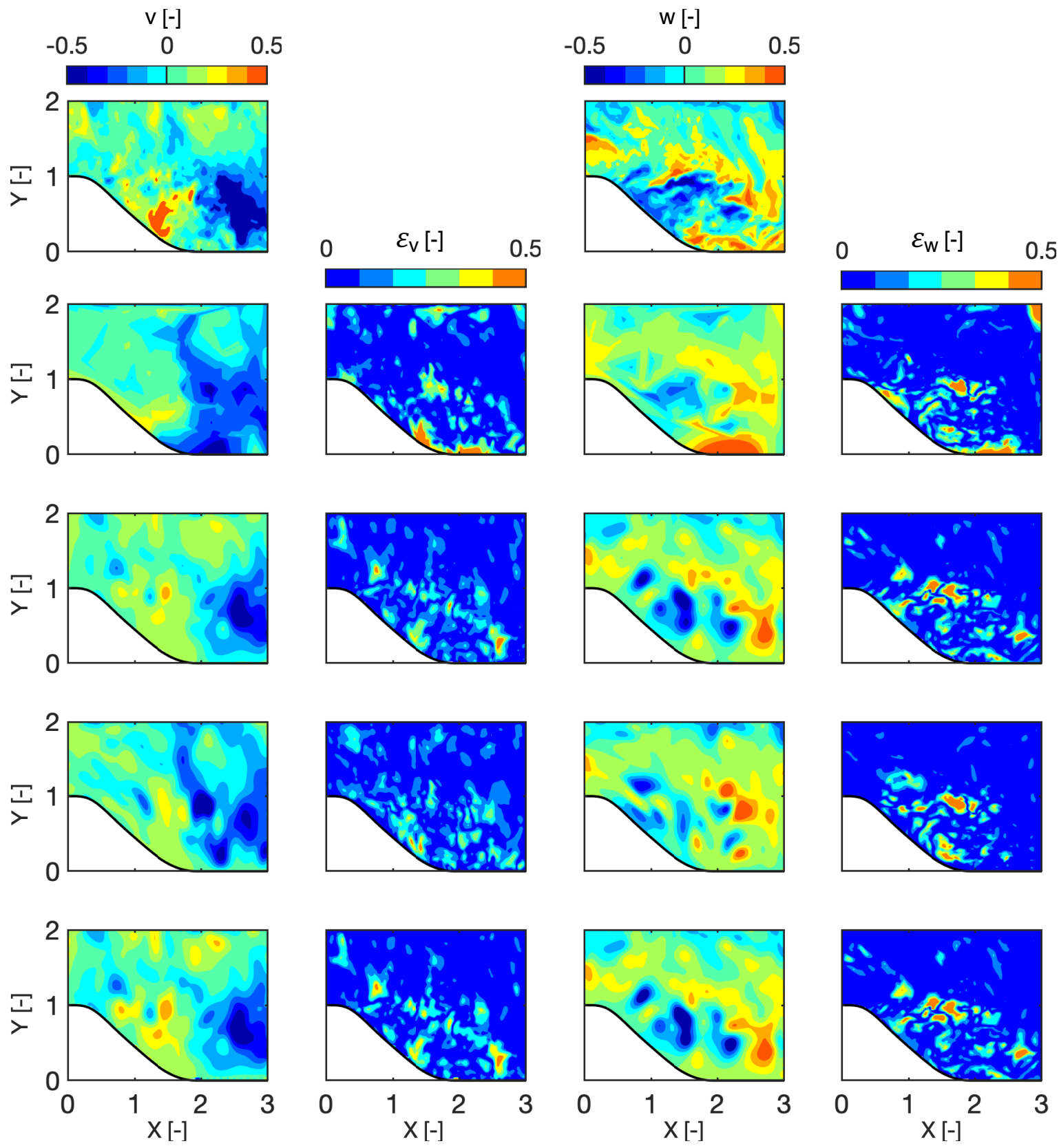

Figure 4: Instantaneous non-dimensional normal (left) and spanwise (right) velocity distribution over the $Z=0$ plane for the reference DNS results ( $1^{\text {st }}$ row). Instantaneous non-dimensional normal ( $I^{\text {st }}$ column) and spanwise ( $3^{\text {rd }}$ column) velocity distribution over the $Z=0$ plane with tri-linear interpolation, VIC + , $A L E-V I C+$ and ImVIC $+\left(2^{\text {nd }}\right.$ to $5^{\text {th }}$ rows $)$. Planar distributions of non-dimensional normal ( $1^{\text {st }}$ column) and spanwise ( $3^{\text {rd }}$ column) velocity reconstruction error magnitudes over the $Z=0$ plane with tri-linear interpolation, VIC+, ALE-VIC+ and ImVIC+ (2 $2^{\text {nd }}$ to $5^{\text {th }}$ rows $)$. 
of velocity and acceleration values, the particle tracks are reconstructed with 7 particles. Then a Gaussian noise of 0.2 voxels in all three dimensions are artificially introduced to particle locations along the tracks to account for measurement and reconstruction errors. Finally, the corresponding particle tracks are regularized with polynomials of order 2 to compute velocity and material acceleration properties. Moreover, in order to model the scarcity of particles in the near wall region observed in experimental data sets, particle tracks within the close proximity of the wall are removed from the synthetic data (Schneiders et al., 2017). To be able to demonstrate the improvements obtained with the proposed approaches over the state of the art of data assimilation, the standard VIC+ method is also employed to perform the dense interpolation of the velocity and material accelerations. However, since the base algorithm of VIC+ is not equipped with a capability of handling the non-uniform boundary, the grid locations corresponding to the solid domain are artificially modified to have zero velocity and material acceleration values to prevent numerical divergence of the optimization procedure.

To start with the pure PTV approach where the AGW is used to capture flow properties in close surroundings of each grid locations, the results failed to provide almost any fluidic information due to the lack of particles. Hence, the corresponding need for an interpolation approach to reconstruct a coherent flow field description is addressed by linear interpolation, VIC+, ALE-VIC+ and ImVIC+ approaches. The qualitative comparisons of velocity magnitude distributions reveals similar characteristics of overall coherence levels with the reference simulations in terms of identifying major flow structures (Fig. 3, $2^{\text {nd }}$ row). Accordingly, the reconstructed streamwise velocity components using both linear interpolation and VIC+ variants represent an accelerated flow behavior over the hill form (Fig. 3. A) and a separation region with recirculating flow downstream (Fig. 3, B). Nevertheless, the detailed structures of local velocity magnitude variations are depicted with increased accuracy using VIC+ variants as the separation effects are captured with greater agreement to the reference (Fig. 3, A \& B). Furthermore, the major differences between the original VIC+ and proposed variants are observed especially in close proximity of the hill surface. The peak fluctuation magnitudes are resolved with a greater agreement to the reference simulations by employing both ALE-VIC+ and ImVIC+ whereas the independency of VIC+ from the relevant surface shape caused loss of accuracy close to the interaction interface that also propagated towards the regions away from the surface (Fig. 3, $3^{\text {rd }}$ row).

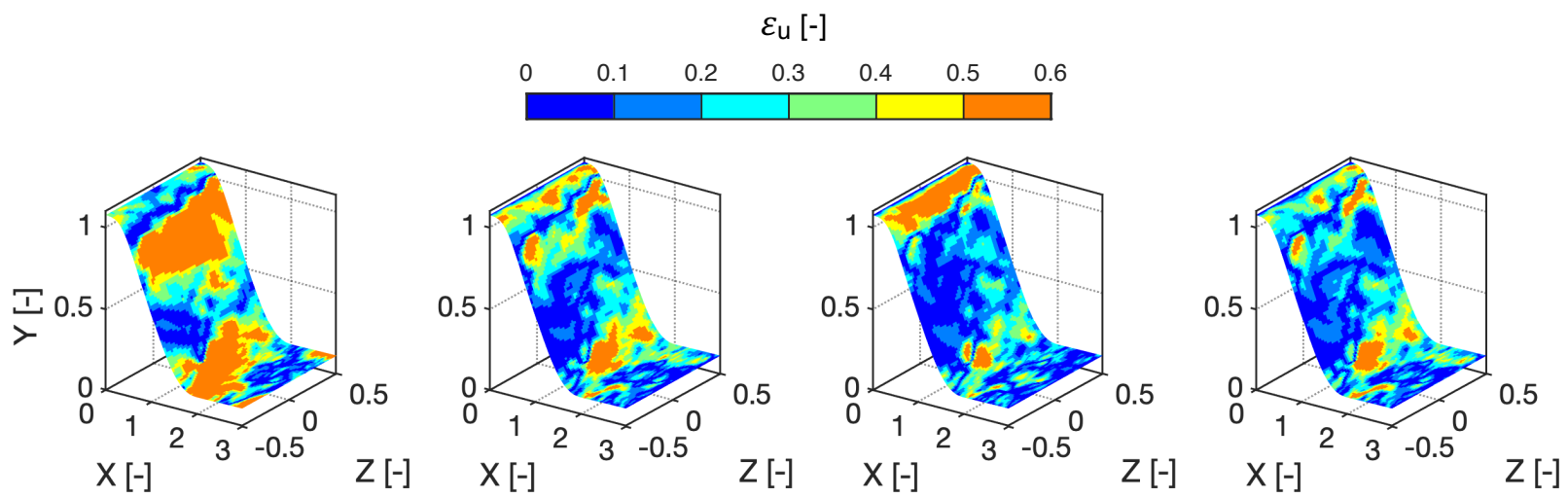

Figure 5: Planar distributions of non-dimensional streamwise velocity reconstruction error magnitudes at 0.1 of the hill surface with tri-linear interpolation, VIC+, ALE-VIC+ and ImVIC+ (from left to right).

Moreover, the superiority of VIC+ variants over linear interpolation becomes even more apparent when the comparisons are performed over the secondary velocity components of normal and spanwise flow elements. In absence of a dominant flow behavior enforced by the immersed boundary form, reconstructions of secondary flow structures represent the ability of VIC+ based approaches to resolve the fluidic behavior with greater detail by means of appropriate physical definitions (Fig. 4, $1^{\text {st }}$ and $3^{\text {rd }}$ columns). The major differences between the original VIC+ method and the proposed variants are again observed in close proximity of the hill surface. Both ALE-VIC+ and ImVIC+ approaches provided reduction in reconstruction errors in comparison to reference data. However, the corrections due to immersed boundary treatment implemented by ImVIC+ have no influence on the spanwise velocity magnitudes (Fig. 4, $3^{\text {rd }}$ and $4^{\text {th }}$ columns). Considering the orientation of the surface elements designated for the hill form form, the fluid penetration through the hill surface is caused by the streamwise and normal velocity vector components. Accordingly, the application ImVIC+ provides modifications to the flow properties within those directions to prevent unphysical 
flow peneration through the surface by ensuring the satisfaction of no-through boundary condition. Hence, the resultant superposition of potential and rotational velocity fields does not impose any alterations on the spanwise velocity magnitudes which can be depicted when compared against the results of VIC+.

Finally, the reduction in error levels gives further evidence of the improved accuracy with the application of ALE-VIC+ and ImVIC+, compared to both linear interpolation and the standard VIC+. Moreover, the dominant effect of the immersed boundary treatment is observed for the streamwise velocity components where decreased error levels in close proximity of the periodic hill surface refer to an elevated level of accuracy over the original VIC+ application with the immersed boundary treatment (Fig. 5). Nonetheless, mitigation of error levels in close proximity of the hill surface denote improved accuracy of the dense flow field reconstructions as a result of the modifications implemented by ImVIC+. The observed modulations between the different methods tend to decrease significantly in regions away from the hill surface which is consistent with the theoretical formulations of surface singularities where the influence of singularity elements decay with the Euclidean distance (Fig. $3,3^{\text {rd }}$ row).

\section{Experimental Assessment}

As a part of the Holistic Optical Metrology for Aero-Elastic Research (HOMER) European Union H2020 project, the experimental setup employed in this work is designed to investigate fluid-structure kinematics of turbulent boundary layer-unsteady panel interactions where the experiments are conducted in a low-speed wind tunnel of TU Delft High Speed Laboratory at a freestream velocity of $10 \mathrm{~m} / \mathrm{s}$. The panel to be deformed is a square elastic membrane with sufficiently high moment of inertia to prevent any aeroelastic deformations and have full control over the membrane shape. A DC motor is connected to the center of the membrane by means of a gear and rod mechanism, and actuated at three different frequencies of $1 \mathrm{~Hz}, 3 \mathrm{~Hz}$ and $5 \mathrm{~Hz}$ with an amplitude of $30 \mathrm{~mm}$ from valley to crest. The corresponding reduced frequencies obtained with the membrane motion of $1 \mathrm{~Hz}$ and $3 \mathrm{~Hz}$ are in the order of $k \sim 0.05$ where a quasi-steady state can be assumed. However, the reduced frequency at $5 \mathrm{~Hz}$ reaches to a level of $k \approx 0.1$ and shall be considered within the unsteady aerodynamics regime (Leishman, 2016). Furthermore, a black foil with a regular grid of light-grey dots is applied to the upper face of the model to enable the structural displacement measurements by means of Lagrangian particle tracking (LPT). A $120 \mathrm{~cm}$ long rigid plate with lego blocks located at $10 \mathrm{~cm}$ from its leading edge is installed upstream of the model to ensure well-developed turbulent boundary conditions at the test section. The flow is seeded with HFSB tracers, which are inserted into the flow in the wind tunnel's settling chamber via an in-house built seeding rake at rate of $2 \times 10^{6}$ bubbles/second. The image acquisiton is performed with three Photron FastCAM SA1.1 CMOS cameras while the measurement volume is illuminated by means of three LED light sources (LaVision LED-flashlight 300). Finally, the experimental setup is equipped with two TruStability board mount pressure sensors to provide reference static pressure values for comparisons against the results of non-intrusive surface pressure reconstruction algorithms.

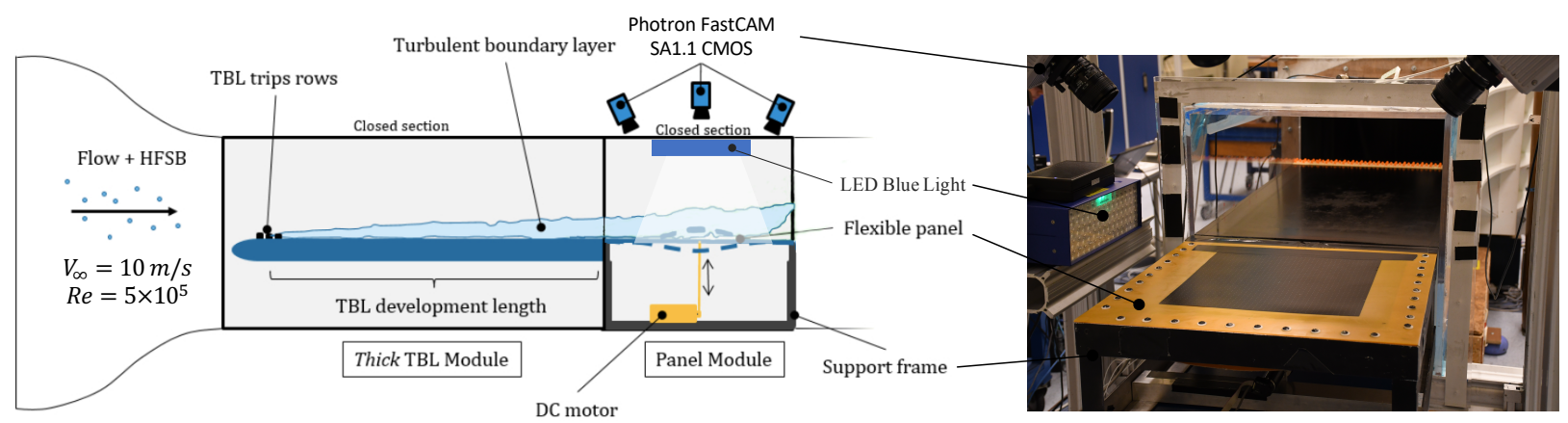

Figure 6: Schematic representation (left) and photo (right) of the experimental setup for turbulent boundary layer interactions with unsteadily deforming elastic membrane.

The acquired images are processed with the STB algorithm for LPT (Schanz et al., 2016) using the commercial software package DaVis version 10.0.5 from LaVision GmbH. Then the particle tracks are reconstructed with a minimum length of 7 particles within consecutive images. For the computation of velocity and Lagrangian acceleration information, a minimum number of 5 particles are selected for fitting a 3rd order polynomial for regularization of the particle motions in the temporal domain. As the membrane 
surface is equipped with tracer markers, surface markers possessed greater light intensities captured by the recording devices compared to the HFSB tracers of the fluid domain. Therefore, sequential employment of low-pass and high-pass filters allowed the individual analysis of fluid and solid tracers respectively. The employed large-scale experimental setup resulted in a tracer particle concentration of $C=100 \mathrm{par} / \delta_{99}$ where the thickness of the fully developed turbulent boundary layer just upstream of the membrane was recorded as $\delta_{99}=0.06 \mathrm{~m}$. Hence, with the corresponding image particle density of $N_{P}=0.02 \mathrm{ppp}$, using the raw STB data becomes inadequate for capturing any relevant flow features. Therefore, trilinear interpolation, VIC+, ALE-VIC+ and ImVIC+ approaches are employed to increase the spatial resolution of fully time-resolved flow field information.

Furthermore, in order to enable a comparative analysis with the pressure tap readings, the pressure field over the densely interpolated flow field data is computed by relating the material accelerations to the static pressure distribution over the Navier-Stokes equation. The resulting pressure gradients are integrated by constructing a Poisson equation while neglecting the viscous diffusion terms due to the low order of magnitude, $O\left(10^{-5}\right)$, of their influence for turbulent flow conditions.

$$
\nabla^{2} p=\nabla \cdot(\nabla p)=\nabla \cdot\left(-\rho \frac{D \mathbf{u}}{D t}\right)
$$

Then, the reconstruction of surface pressure over the non-uniformly deformed elastic membrane is performed utilizing two different approaches of an omni-directional integration procedure similar to the approach introduced by Jux et al. (2020) and a reconstruction scheme with curvilinear transformations proposed by Cakir (2020). The letter approach enabled direct computation of the surface pressure alongside the global pressure information without the need of external extrapolations.
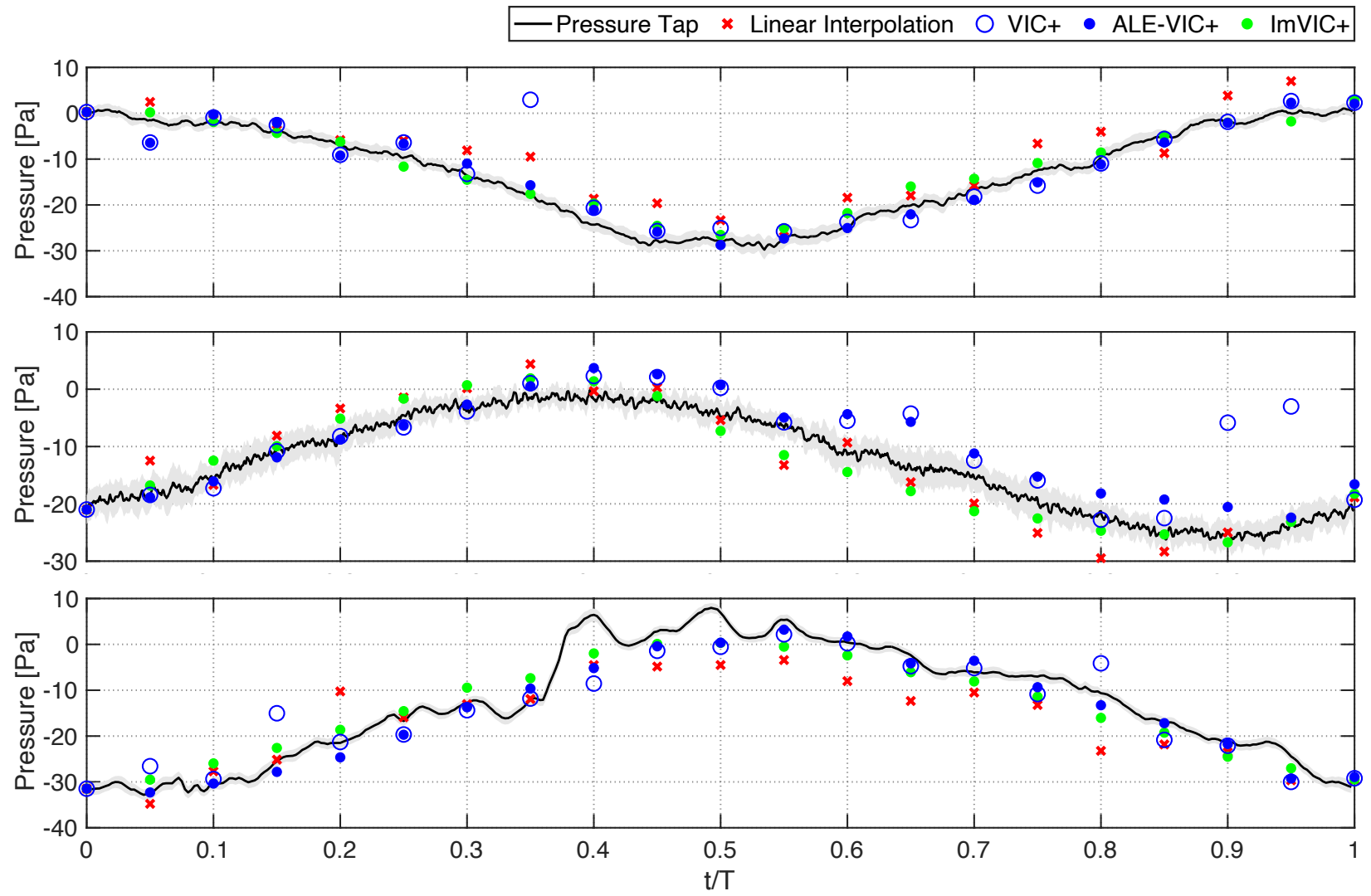

Figure 7: Instantaneous pressure reconstruction of linear interpolation, VIC+, ALE-VIC+ and ImVIC+ methods in comparison to the pressure tab measurements for $1 \mathrm{~Hz}$ (top), $3 \mathrm{~Hz}$ (middle) and $5 \mathrm{~Hz}$ (bottom) of unsteady membrane motion.

Comparing the surface pressure information reconstructed with four different approaches, the variations throughout the overall pressure profiles are observed to be relatively confined. Nevertheless, both 
ALE-VIC+ and ImVIC+ enabled a greater agreement with the pressure tap measurements by minor improvements (Fig. 7). Three main reasons can be deduced for these minor variations in correlation with the validation studies. First of all, the spatial gradients of both velocity and pressure over the measurement domain are dominated by the controlled motion of the elastic membrane. Hence, as motion frequencies of the membrane correspond to reduced frequencies of $k<0.1$, these gradients can be reconstructed even with the linear interpolation without severe loss of accuracy. Secondly, considering the assumption of zero pressure gradients within the turbulent boundary layers and the membrane motion, the Dirichlet boundary condition dictates the high percentage of pressure variations in time. Although there exists non-zero pressure gradients throughout the realistic boundary layers, these relate to pressure modulations in the order of $6 \sim 8 \mathrm{~Pa}$ while the absolute pressure difference captured at highest deformation state of the membrane is $\sim 30 \mathrm{~Pa}$. Hence, the pressure variations aimed to be reconstructed with a superior accuracy by ALE-VIC+ and ImVIC+ correspond to $\sim 25 \%$ of the pressure modulation amplitude. Lastly, the boundary conditions for VIC+, ALE-VIC+ and ImVIC+ methods are determined via linearly interpolating the STB data over the corresponding boundaries. Considering the close proximity of the Dirichlet boundary condition of pressure reconstruction to the computational domain boundaries of VIC+ variants, the differences between the various approaches in terms of the absolute pressure values are further alleviated.

Table 1: RMS of instantaneous pressure reconstruction errors [Pa] of linear interpolation, VIC+, ALE-VIC+ and ImVIC+ for $1 \mathrm{~Hz}, 3 \mathrm{~Hz}$ and $5 \mathrm{~Hz}$ of unsteady membrane motion.

\begin{tabular}{lccc}
\hline \hline & \multicolumn{3}{c}{ Motion frequency } \\
\cline { 2 - 4 } & $\mathrm{HHz}$ & $3 \mathrm{~Hz}$ & $5 \mathrm{~Hz}$ \\
\hline Linear Interpolation & 5.2 & 4.7 & 6.8 \\
VIC+ & 4.9 & 5.1 & 5.3 \\
ALE-VIC+ & 2.1 & 2.1 & 4.1 \\
ImVIC+ & 2.1 & 2.1 & 3.6 \\
\hline \hline
\end{tabular}

Nonetheless, there exits specific time instants where the differences between VIC+ and the proposed variants are amplified with reconstruction errors up to $\sim 70 \%$ of the pressure fluctuation amplitudes obtained using VIC+. This is related to the extreme sensitivity of the standard VIC+ method to the spatial distribution of the available particle tracking information. The absence of a proper boundary condition definition in close proximity of the elastic membrane, VIC+ algorithm becomes strongly dependent on particles in the near wall regions to drive the optimization procedure towards an accurately solution which is a considerably rare situation in practical cases. Therefore, ALE-VIC+ and ImVIC+ approaches provide the capability of accurately reconstructing the flow properties even in the cases of complete absence of particles in the near wall regions by providing a kinematic characterization of the solid boundary intrusions. The resultant accuracy improvements achieved by the implementation of ALE-VIC+ and ImVIC+ over linear interpolation and VIC+ are also observed over the cumulative error levels over the pressure reconstruction profiles (Tab. 1).

\section{Conclusions}

PTV techniques reveal scattered data structures that are required to be fitted on regular grids in order to analyze the measurement results. In case of large scale applications with the use of HFSB tracer particles, reduced particle concentration specifications further depletes the flow characterization capabilities of timeresolved data sets. However, the available governing equation based data assimilation techniques such as FlowFit (Gesemann et al., 2016) and VIC+ Schneiders and Scarano (2016) enable dense volumetric interpolations of flowfield information for regions of uniform rectangular computational domains with sole fluid presence. In this regard, the introduced variants of ALE-VIC+ and ImVIC+ provide the standard VIC+ algorithm with the capability of incorporating appropriate boundary condition definitions for dense flow field characterization in close proximity of solid objects with generic geometries. ALE-VIC+ method implements the ALE method with an RBF based mesh deformation scheme to ensure the adaptability of the grid formations to the unsteady deformations of the FSI interface. On the other hand, ImVIC+ approach preserves FFT based Poisson solvers to increase computational efficiency using uniform predefined computational grids where immersed boundary treatments are utilized to satisfy the boundary conditions by means of additional flow components. 
The validation studies of the proposed methods are performed with a numerical test case of flow over periodic hills, where the DNS data sets are manipulated to simulate realistic experimental conditions. Even though both linear interpolation and VIC+ variants resulted in coherent flow behaviors with the hill form, the local variations of velocity components favored the latter in terms of greater reconstruction accuracy. With the application of ALE-VIC+ and ImVIC+, reconstruction accuracy improvements over the standard VIC+ method are achieved especially in close proximity of the hill surfaces in terms of streamwise and normal velocity components composing the surface flow penetrations. The modifications are observed to be confined to the close proximity of the solid boundaries where the particle tracking information is able to construct an accurate objective function for the optimization procedure.

Finally, both data assimilation approaches are applied to enable instantaneous flow field characterization for the measurements of turbulent boundary layer interactions with a dynamically deforming elastic membrane. The densely reconstructed flow field properties are then employed to compute the pressure distribution over membrane surface, revealing the time-resolved interaction between the flow structures and the membrane deformations. The superior accuracy specifications of ALE-VIC+ and ImVIC+ methods against trilinear interpolation and the standard VIC+ method achieved by enabling the kinematic discretization of the unsteadily deforming membrane motion, provided a greater agreement with the pressure tap measurements by reducing surface pressure reconstruction errors by $\sim 50 \%$ for all three membrane motion frequencies.

\section{Acknowledgements}

This work has been carried out in connection to the project HOMER (Holistic Optical Metrology for AeroElastic Research), which is funded by the European Commission, program H2020 under Grant No. 769237. Also, the authors would like to thank Prof. Stefan Hickel from Delft University of Technology for providing the periodic hill flow DNS data set that is used for the numerical assessments.

\section{References}

Agui JC and Jimenez J (1987) On the performance of particle tracking. Journal of Fluid Mechanics $185: 447-468$

Beale JT and Greengard C (1994) Convergence of euler-stokes splitting of the navier-stokes equations. Communications on Pure and Applied Mathematics 47:1083-1115

Beckert A and Wendland H (2001) Multivariate interpolation for fluid-structure-interaction problems using radial basis functions. Aerospace Science and Technology 5:125 - 134

Cakir BO (2020) Bridging Experimental Simulations with Computational Frameworks for Time-Resolved Characterization of Fluid-Structure Interactions. Master's thesis. Delft University of Technology

Caridi GCA, Ragni D, Sciacchitano A, and Scarano F (2016) HFSB-seeding for large-scale tomographic PIV in wind tunnels. Experiments in Fluids 57:190

Casa LDC and Krueger PS (2013) Radial basis function interpolation of unstructured, three-dimensional, volumetric particle tracking velocimetry data. Measurement Science and Technology 24:065304

Chen ZL, Hickel S, Devesa A, Berland J, and Adams NA (2014) Wall modeling for implicit large-eddy simulation and immersed-interface methods. Theor Comput Fluid Dyn 28:1-21

Christiansen I (1973) Numerical simulation of hydrodynamics by the method of point vortices. Journal of Computational Physics 13:363 - 379

Cottet GH and Koumoutsakos PD (2000) Hybrid methods. in Vortex Methods: Theory and Practice. page 237-260. Cambridge University Press

Cottet GH and Poncet P (2004) Advances in direct numerical simulations of 3d wall-bounded flows by vortex-in-cell methods. Journal of Computational Physics 193:136 - 158

de Boer A, van der Schoot M, and Bijl H (2007) Mesh deformation based on radial basis function interpolation. Computers \& Structures 85:784 - 795 
Dowell EH (2004) Modeling of fluid-structure interaction. in A Modern Course in Aeroelasticity. pages 491-539. Springer Netherlands

Elsinga G, Scarano F, Wieneke B, and Oudheusden B (2006) Tomographic particle image velocimetry. Experiments in Fluids 41:933-947

Farhat C, Degand C, Koobus B, and Lesoinne M (1998) Torsional springs for two-dimensional dynamic unstructured fluid meshes. Computer Methods in Applied Mechanics and Engineering 163:231 - 245

Fukuchi Y (2012) Influence of number of cameras and preprocessing for thick volume Tomographic PIV. in $16^{\text {th }}$ Int. Symp. on Applications of Laser Techniques to Fluid Mechanics, July 9-12, 2012. Lisbon, Portugal

Gesemann S, Huhn F, Schanz D, and Schröder A (2016) From noisy particle tracks to velocity, acceleration and pressure fields using b-splines and penalties. in $18^{\text {th }}$ International Symposium on the Application of Laser and Imaging Techniques to Fluid Mechanics, July 4-7, 2016. Lisbon, Portugal

Helenbrook BT (2003) Mesh deformation using the biharmonic operator. International Journal for Numerical Methods in Engineering 56:1007-1021

Hess J and Smith A (1967) Calculation of potential flow about arbitrary bodies. Progress in Aerospace Sciences 8:1- 138

Hwang T, Doh D, Jo H, Tsubokura M, Piao B, Kuroda S, Kobayashi T, Tanaka K, and Takei M (2007) Analysis of fluid-elastic-structure interactions in an impinging jet with a dynamic 3D-PTV and noncontact 6D-motion tracking system. Chemical Engineering Journal 130:153 - 164

Jones W and Samareh-Abolhassani J (2012) A grid generation system for multi-disciplinary design optimization. in AIAA $12^{\text {th }}$ Computational Fluid Dynamics Conference, June 19-22, 1995. San Diego, CA, U.S.A.

Jux C, Sciacchitano A, and Scarano F (2020) Flow pressure evaluation on generic surfaces by robotic volumetric PTV. Measurement Science and Technology 31:104001

Katz J and Plotkin A (2001) Low-Speed Aerodynamics. Cambridge Aerospace Series. Cambridge University Press. $2^{\text {nd }}$ edition

Kudela H and Kozlowski T (2009) Vortex in cell method for exterior problems. Journal of Theoretical and Applied Mechanics 47:779-796

Leishman J (2016) Principles of Helicopter Aerodynamics. Cambridge Aerospace Series. Cambridge University Press

Lewis RI (1991) The basis of surface singularity modelling. in Vortex Element Methods for Fluid Dynamic Analysis of Engineering Systems. Cambridge Engine Technology Series. page 3-43. Cambridge University Press

Liu X, Qin N, and Xia H (2006) Fast dynamic grid deformation based on delaunay graph mapping. Journal of Computational Physics 211:405 - 423

Löhner R and Yang C (1996) Improved ale mesh velocities for moving bodies. Communications in Numerical Methods in Engineering 12:599-608

Luke E, Collins E, and Blades E (2012) A fast mesh deformation method using explicit interpolation. Journal of Computational Physics 231:586 - 601

Morgenthal G and Walther J (2007) An immersed interface method for the vortex-in-cell algorithm. Computers \& Structures 85:712-726

Noh WF (1963) CEL: A time-dependent, two-space dimensional, coupled Eulerian-Lagrange code. Technical Report UCRL-7463. Lawrence Radiation Lab., Univ. of California. Livermore, CA, U.S.A

Peskin C (1982) The fluid dynamics of heart valves: Experimental, theoretical, and computational methods. Annual Review of Fluid Mechanics 14:235-259 
Sagredo B and Tercero JCO (2003) Z-splines: moment conserving cardinal spline interpolation of compact support for arbitrarily spaced data. Research Report / Seminar für Angewandte Mathematik 2003-10

Scarano F, Ghaemi S, Caridi GCA, Bosbach J, Dierksheide U, and Sciacchitano A (2015) On the use of helium-filled soap bubbles for large-scale tomographic piv in wind tunnel experiments. Experiments in Fluids 56:42

Schanz D, Gesemann S, and Schröder A (2016) Shake-the-box: Lagrangian particle tracking at high particle image densities. Experiments in Fluids 57:1-27

Schneiders J, Azijli I, Scarano F, and Dwight R (2015) Pouring time into space. in $11^{\text {th }}$ International Symposium on Particle Image Velocimetry, September 14-16, 2015. Santa Barbara, CA, U.S.A.

Schneiders JFG and Scarano F (2016) Dense velocity reconstruction from tomographic PTV with material derivatives. Experiments in Fluids 57:139

Schneiders JFG, Scarano F, and Elsinga GE (2017) Resolving vorticity and dissipation in a turbulent boundary layer by tomographic PTV and VIC+. Experiments in Fluids 58:27

Smith MJ, Cesnik CES, and Hodges DH (2000) Evaluation of some data transfer algorithms for noncontiguous meshes. Journal of Aerospace Engineering 13:52-58

Soifer V (2013) Main equations of diffraction theory. in Computer Design of Diffractive Optics. Woodhead Publishing Series in Electronic and Optical Materials. pages 1 - 24. Woodhead Publishing

Tarafder S, Saha G, and Sayeed T (2010) Analysis of potential flow around 3-dimensional hydrofoils by combined source and dipole based panel method. Journal of Marine Science and Technology 18:376-384

Tokarev MP, Alekseenko MV, Bilsky AV, Dulin VM, and Markovich DM (2013) Tomographic PIV measurements in a swirling jet flow. in $10^{\text {th }}$ International Symposium on Particle Image Velocimetry - PIVI3, July 2-4, 2013. Delft, The Netherlands

Walther JH and Morgenthal G (2002) An immersed interface method for the vortex-in-cell algorithm. Journal of Turbulence 3:N39

Wang Z and Przekwas A (2012) Unsteady flow computation using moving grid with mesh enrichment. in AIAA $32^{\text {nd }}$ Aerospace Sciences Meeting and Exhibit, January 10-13, 1994. Rano, NV, U.S.A.

Wu J and JaJa J (2013) High performance FFT based poisson solver on a CPU-GPU heterogeneous platform. in IEEE $27^{\text {th }}$ International Parallel and Distributed Processing Symposium - IPDPS 2013, May 20-14, 2013. pages 115-125. Boston, MA, U.S.A 